\title{
Evaluation of chromium, nickel, iron and manganese content in wheat, flour, bran and selected baked products
}

\author{
Piotr Bawiec ${ }^{1}$, Magdalena Halabis ${ }^{\star 2}$, Zbigniew Marzec ${ }^{1}$, Andrzej Kot ${ }^{1}$, \\ JANUSZ SOLSKI ${ }^{2}$, KInga GAWEL ${ }^{3}$

\begin{abstract}
${ }^{1}$ Chair and Department of Food and Nutrition, Medical University of Lublin, Chodzki 4a, 20-093 Lublin, Poland
${ }^{2}$ Department of Laboratory Diagnostics, Medical University of Lublin, Chodzki 1, 20-093 Lublin, Poland

${ }^{3}$ Chair and Department of Pharmacology and Pharmacodynamics, Medical University of Lublin, Chodzki 4a, 20-093 Lublin, Poland
\end{abstract}

\begin{tabular}{l}
\hline ARTICLE INFO \\
\hline Received 23 April 2014 \\
Accepted 30 May 2014 \\
\hline
\end{tabular}

\section{KEYWORDS:}

trace elements,

chromium,

nickel,

iron,

manganese,

bread.

\begin{abstract}
Considering the nutritional values, breadstuff plays a big part in covering human nourishment needs and constitutes a base of all day diet. Moreover, bread is an excellent source of numerous vitamins and minerals the abundance of which depends on the degree of grinding. Thus, it seems to be very important to know the composition and level of bio-elements. That is why the main target of this study was to evaluate the concentration of selected trace elements: chromium $(\mathrm{Cr})$, nickel $(\mathrm{Ni})$, iron $(\mathrm{Fe})$ and manganese $(\mathrm{Mn})$ in wheat grain, wheat bran, different wheat and rye flour types and variety of breadstuff also with addition of grains and seeds from different bakeries and mills. Another task was to analyze if the technological process has an influence on secondary despoil of bread goods with heavy metal elements. The analyzed trace elements were measured with a precise and accurate atomic absorption spectrophotometric method (AAS) and the results were expressed in $\mathrm{mg} / \mathrm{kg}$ of selected sample. Obtained results show that bread and grain products are a good source of trace elements like chromium, nickel, iron and manganese. However, the higher levels of chromium and nickel in bread goods could rather be an effect of impurity caused by a technological process in mill and bakeries.
\end{abstract}

\section{INTRODUCTION}

The actual healthy diet is based on natural goods like grains and their products. Grain products like breadstuff comprise about $80 \%$ of adult's daily diet in Poland. Estimations show that a statistical Pole consumes about $4.46 \mathrm{~kg}$ of bread each month and prefers rather white bread $[4,14]$.

It is assumed that the grain products should cover daily human need on energy in $50 \%$, proteins in $35 \%$, calcium in $25 \%$, iron in $50 \%$ and vitamin B in $40 \%$. Considering the nutrient values breadstuff plays a big role in covering human nourishment needs and constitutes a base of all day diet. Moreover, bread is an excellent source of numerous vitamins and minerals the abundance of which depends on the degree of grinding. Thus, it seems to be very important to know the composition and level of bio-elements $[2,5,6]$.

\section{Corresponding author}

e-mail: magdalenahalabis@gmail.com

tel/fax. +48 81 742-38-13

cell phone: +48691969101
According to the present expectations of society, bakeries enhance the production of a variety of bread goods enriched with natural additives like grains and seeds, which have significant nourishing influence and also because of losses in nutrients occurring in the process of grinding [11].

Cereal products including breadstuff and bread are not free from the impurities. Such substances like trace elements are controversial because their trivalent forms are necessary for a proper functioning of the organism. On the other hand, such compounds in high levels are toxic and carcinogenic. In industry, bio-elements are popular, therefore their pollutes in soil and water, following accumulates in plant tissues and present in abnormal values could be hazardous for human health in a significant way. Thus, while analyzing the level of food impurity it should be taken into account that the additional substances like trace elements could result from the natural chemical content of food as well as from the environmental pollution with the specific elements [12,13].

The main target of this study was to evaluate the concentration of selected trace elements: chromium $(\mathrm{Cr})$, nickel $(\mathrm{Ni})$, iron (Fe) and manganese (Mn) in wheat grain, wheat 
bran, different wheat and rye flour types and variety of breadstuff also with addition of grains and seeds.

Another aim of this study was to show the relationship between the level of heavy metals in samples and the type of bakery; thus, the samples were taken from three places: „Kolstar” bakery in Lublin, „Marysin” bakery in Marysin and mill in Dys near Lublin.

The last task was to analyze if the technological process has an influence on secondary despoil of bread goods with heavy metal elements.

\section{MATERIAL AND METHODS}

The studied material comprised:

1. Six types of bread: sliced mixed, whole-meal rye mixed, Magnus, bread with sunflower seed, graham bread with spelt wheat and rye bread; four kinds of wheat bread stuff like graham rolls (I and II) and fancy rolls (I and II); two types of wheat flour (type 500 and 750); two types of rye flour (type 720 and 2000) and graham flour type 1950. Samples were taken from the bakery "Kolstar" in Lublin.

2. Seven types of bread: grainy, rye, "Gwarek", Slavic, pastoral, manufacturer sliced mixed bread and multigrain. Six types of breadstuff like fancy roll, fancy roll with powder, graham roll, graham roll with grains, Turkish roll with raisins and sweet Turkish roll. Five types of wheat flour type: 500, 550, 650, 750, 1850; two types of rye flour: 720, 2000; bran and "Gwarek" mixture. The samples were taken from the bakery "Marysin" from Marysin.

3. Eight samples of: wheat grain, wheat flour and wheat bran from mill in Dys near Lublin.

For the analysis, we collected 2-4 pieces of each bread goods for making an average sample. Twenty-grams parallel samples in a number 5-10 were weighed out from the average sample and used for the instrumental analysis. Samples were dried and burnt to ash in a muffle furnace in $450^{\circ} \mathrm{C}$. The standard solutions were prepared from the named solutions:

- chromium standard - Chrom - Standardlösung, $\mathrm{CrCl}_{3}$ in verd. Salzsäure, by Merck, containing $1 \mathrm{~g} \pm 0.002 \mathrm{~g} \mathrm{Cr}$ in $1 \mathrm{dm}^{3}$ of solution,

- nickel standard - Nickel - Standardlösung, $\mathrm{NiCl}_{2}$ in Wasser, by Merck, containing $1 \mathrm{~g} \pm 0.002 \mathrm{~g} \mathrm{Ni}$ in $1 \mathrm{dm}^{3}$ of solution,

- iron standard - Eisen - Standardlösung, $\mathrm{FeCl}_{3}$ in verd. Salzsäure, by Merck, containing $1 \mathrm{~g} \pm 0.002 \mathrm{~g}$ Fe in $1 \mathrm{dm}^{3}$ of solution,

- manganese standard - Mangan - Standardlösung, by Merck, containing $1 \mathrm{~g} \pm 0.002 \mathrm{~g} \mathrm{Mn}$ in $1 \mathrm{dm}^{3}$ of solution.

From the given standard solution, the working solutions were prepared containing $1 \mu \mathrm{g}$ of nickel, chromium, iron and manganese in $\mathrm{cm}^{3}$. Having made the standard calibration curves, we measured absorbance of chromium, nickel, iron and manganese in spectrophotometer Pye Unicam Sp - 192 or Solaar M5.

The chromium, nickel, iron and manganese concentrations were measured with a precise and accurate atomic absorption spectrophotometric method (AAS) and the results were expressed in $\mathrm{mg} / \mathrm{kg}$.

The obtained results were statistically analyzed. Arithmetic mean values and standard deviation (SD) were calculated with the use of STATISTICA 6.0 software.

\section{RESULTS AND DISCUSSION}

The content of chromium, nickel, iron and manganese in flour, wheat and bran derived from the same mill in Dys is reported in Table 1. The flour and bran obtained from homogenous wheat grain were analyzed to find out how the trace elements were spread in grain. The highest concentrations of chromium $(0.197 \mathrm{mg} \mathrm{Cr} / \mathrm{kg})$, nickel (0.292 $\mathrm{mg} \mathrm{Ni} / \mathrm{kg})$, iron (53.89 $\mathrm{mg} \mathrm{Fe} / \mathrm{kg}$ ) and manganese (48.00 mg Mn/kg) were observed in bran. The level of chromium was about twice- and nickel about four-times higher in bran than flour. The iron and manganese are spread in the external layer of grain thus the concentration of iron was five-times and manganese eight-times higher in bran than flour (Table 1).

Table 1. Chromium (Cr), nickel (Ni), iron (Fe) and manganese $\mathrm{Mn})$ content in flour, wheat and bran from mill in Dys

\begin{tabular}{|c|c|c|c|c|c|}
\hline Product & Location & $\begin{array}{l}\mathrm{Cr}[\mathrm{mg} / \mathrm{kg}] \\
\text { mean } \pm \mathrm{SD}\end{array}$ & $\begin{array}{l}\mathrm{Ni}[\mathrm{mg} / \mathrm{kg}] \\
\text { mean } \pm \mathrm{SD}\end{array}$ & $\begin{array}{l}\mathrm{Fe}[\mathrm{mg} / \mathrm{kg}] \\
\text { mean } \pm \mathrm{SD}\end{array}$ & $\begin{array}{c}\mathrm{Mn}[\mathrm{mg} / \mathrm{kg}] \\
\text { mean } \pm \mathrm{SD}\end{array}$ \\
\hline Flour & $\begin{array}{l}\text { Mill in } \\
\text { Dys }\end{array}$ & $\begin{array}{r}0.079 \\
\pm 0.006\end{array}$ & $\begin{array}{r}0.079 \\
\pm 0.024\end{array}$ & $\begin{array}{r}11.26 \\
\pm 1.91\end{array}$ & $\begin{array}{r}5.18 \\
\pm 0.75\end{array}$ \\
\hline Wheat & $\begin{array}{l}\text { Mill in } \\
\text { Dys }\end{array}$ & $\begin{array}{r}0.136 \\
+0.016\end{array}$ & $\begin{array}{r}0.165 \\
\pm 0.074\end{array}$ & $\begin{array}{r}28.21 \\
\pm 2.61\end{array}$ & $\begin{array}{r}25.23 \\
\pm 3.86\end{array}$ \\
\hline Bran & $\begin{array}{l}\text { Mill in } \\
\text { Dys }\end{array}$ & $\begin{array}{r}0.197 \\
\pm 0.032\end{array}$ & $\begin{array}{r}0.292 \\
\pm 0.126\end{array}$ & $\begin{array}{r}53.89 \\
\pm 14.40\end{array}$ & $\begin{array}{r}48.00 \\
\pm 12.25\end{array}$ \\
\hline
\end{tabular}

In Table 2, the concentrations of analyzed trace elements in rye flour were reported. The levels of chromium and nickel were the lowest in rye flour type 720 from Marysin $(0.118 \mathrm{mg} \mathrm{Cr} / \mathrm{kg}$ and $0.107 \mathrm{mg} \mathrm{Ni} / \mathrm{kg})$ and the highest in whole meal rye flour type 2000 from Marysin too $(0.195 \mathrm{mg}$ $\mathrm{Cr} / \mathrm{kg}$ and $0.167 \mathrm{mg} \mathrm{Ni} / \mathrm{kg}$ ). The iron and manganese concentrations correlated with grinding. Their levels varied from $14.32 \mathrm{mg} \mathrm{Fe} / \mathrm{kg}$ and $12.53 \mathrm{mg} \mathrm{Mn} / \mathrm{kg}$ in rye flour type 720 Marysin to $31.72 \mathrm{mg} \mathrm{Fe} / \mathrm{kg}$ and $35.43 \mathrm{mg} \mathrm{Mn} / \mathrm{kg}$ in rye whole meal flour type 2000 from Lublin bakery. However, the concentrations of analyzed elements in selected flours of two different bakeries (Lublin and Marysin) were very similar.

Table 2. Chromium (Cr), nickel (Ni), iron (Fe) and manganese $(\mathrm{Mn})$ content in rye flour from bakery in Lublin and Marysin

\begin{tabular}{|c|c|c|c|c|c|}
\hline Product & Location & $\begin{array}{l}\mathrm{Cr}[\mathrm{mg} / \mathrm{kg}] \\
\text { mean } \pm \mathrm{SD}\end{array}$ & $\begin{array}{l}\mathrm{Ni}[\mathrm{mg} / \mathrm{kg}] \\
\text { mean } \pm \mathrm{SD}\end{array}$ & $\begin{array}{l}\text { Fe }[\mathrm{mg} / \mathrm{kg}] \\
\text { mean } \pm \mathrm{SD}\end{array}$ & $\begin{array}{l}\mathrm{Mn}[\mathrm{mg} / \mathrm{kg}] \\
\text { mean } \pm \mathrm{SD}\end{array}$ \\
\hline \multirow{2}{*}{ Rye flour type 720} & \multirow{2}{*}{ Lublin } & 0.136 & 0.114 & 18.97 & 14.10 \\
\hline & & \pm 0.021 & \pm 0.023 & \pm 1.36 & \pm 0.48 \\
\hline \multirow{2}{*}{ Rye flour type 720} & \multirow{2}{*}{ Marysin } & 0.118 & 0.107 & 14.32 & 12.53 \\
\hline & & \pm 0.035 & \pm 0.032 & \pm 0.67 & \pm 2.57 \\
\hline \multirow{2}{*}{$\begin{array}{l}\text { Rye flour wholemeal } \\
\text { type } 2000\end{array}$} & \multirow{2}{*}{ Lublin } & 0.193 & 0.140 & 31.72 & 35.43 \\
\hline & & \pm 0.007 & \pm 0.021 & \pm 2.14 & \pm 10.45 \\
\hline \multirow{2}{*}{$\begin{array}{l}\text { Rye flour wholemeal } \\
\text { type } 2000\end{array}$} & \multirow{2}{*}{ Marysin } & 0.195 & 0.167 & 28.48 & 30.02 \\
\hline & & \pm 0.076 & \pm 0.060 & \pm 3.56 & \pm 2.50 \\
\hline \multirow{2}{*}{\multicolumn{2}{|c|}{ mean $\pm S D$}} & 0.161 & 0.132 & 23.37 & 23.02 \\
\hline & & \pm 0.039 & \pm 0.027 & \pm 8.11 & \pm 11.44 \\
\hline
\end{tabular}

The content of $\mathrm{Cr}$ in wheat flour (Table 3) varied from $0.104 \mathrm{mg} / \mathrm{kg}$ (type 650 ) to $0.206 \mathrm{mg} / \mathrm{kg}$ (graham type 1950). The lowest concentrations of $\mathrm{Ni}$ and $\mathrm{Mn}$ were observed in wheat flour type 500 from Marysin $(0.094 \mathrm{mg} \mathrm{Ni} / \mathrm{kg}$ 
and $5.17 \mathrm{mg} \mathrm{Mn} / \mathrm{kg}$ ) and the highest in wheat flour type 1850 from Marysin: $0.417 \mathrm{mg} \mathrm{Ni} / \mathrm{kg}$ and $36.04 \mathrm{mg} \mathrm{Mn} / \mathrm{kg}$, respectively. While the iron levels fluctuated from $5.54 \mathrm{mg} / \mathrm{kg}$ (wheat flour type 750) to $38.52 \mathrm{mg} / \mathrm{kg}$ in graham type 1950 .

Table 3. Chromium (Cr), nickel (Ni), iron (Fe) and manganese $(\mathrm{Mn})$ content in wheat flour from bakery in Lublin and Marysin

\begin{tabular}{|c|c|c|c|c|c|}
\hline Product & Location & $\begin{array}{l}\mathrm{Cr}[\mathrm{mg} / \mathrm{kg}] \\
\text { mean } \pm \mathrm{SD}\end{array}$ & $\begin{array}{l}\mathrm{Ni}[\mathrm{mg} / \mathrm{kg}] \\
\text { mean } \pm \mathrm{SD}\end{array}$ & $\begin{array}{l}\mathrm{Fe}[\mathrm{mg} / \mathrm{kg}] \\
\text { mean } \pm \mathrm{SD}\end{array}$ & $\begin{array}{c}\mathrm{Mn}[\mathrm{mg} / \mathrm{kg}] \\
\text { mean } \pm \mathrm{SD}\end{array}$ \\
\hline $\begin{array}{l}\text { Wheat flour type } \\
500\end{array}$ & Lublin & $\begin{array}{r}0.131 \\
\pm 0.024 \\
\end{array}$ & $\begin{array}{r}0.157 \\
\pm 0.143 \\
\end{array}$ & $\begin{array}{r}14.60 \\
\pm 0.58 \\
\end{array}$ & $\begin{array}{r}5.20 \\
\pm 0.30 \\
\end{array}$ \\
\hline $\begin{array}{l}\text { Wheat flour type } \\
500\end{array}$ & Marysin & $\begin{array}{r}0.130 \\
\pm 0.053 \\
\end{array}$ & $\begin{array}{r}0.094 \\
\pm 0.021 \\
\end{array}$ & $\begin{array}{r}12.75 \\
\pm 0.83\end{array}$ & $\begin{array}{r}5.17 \\
\pm 0.40\end{array}$ \\
\hline $\begin{array}{l}\text { Wheat flour type } \\
550\end{array}$ & Marysin & $\begin{array}{r}0.156 \\
\pm 0.009 \\
\end{array}$ & $\begin{array}{r}0.133 \\
\pm 0.023 \\
\end{array}$ & $\begin{array}{r}23.98 \\
\pm 1.58 \\
\end{array}$ & $\begin{array}{r}11.71 \\
\pm 1.01 \\
\end{array}$ \\
\hline $\begin{array}{l}\text { Wheat flour type } \\
650\end{array}$ & Marysin & $\begin{array}{r}0.104 \\
\pm 0.007 \\
\end{array}$ & $\begin{array}{r}0.174 \\
\pm 0.004 \\
\end{array}$ & $\begin{array}{r}22.39 \\
\pm 0.50 \\
\end{array}$ & $\begin{array}{r}12.29 \\
\pm 0.21\end{array}$ \\
\hline $\begin{array}{l}\text { Wheat flour type } \\
750\end{array}$ & Lublin & $\begin{array}{r}0.172 \\
\pm 0.033 \\
\end{array}$ & $\begin{array}{r}0.151 \\
\pm 0.033 \\
\end{array}$ & $\begin{array}{r}28.66 \\
\pm 1.48 \\
\end{array}$ & $\begin{array}{r}13.19 \\
\pm 1.48 \\
\end{array}$ \\
\hline $\begin{array}{l}\text { Wheat flour type } \\
750\end{array}$ & Marysin & $\begin{array}{r}0.187 \\
\pm 0.018 \\
\end{array}$ & $\begin{array}{r}0.141 \\
\pm 0.010 \\
\end{array}$ & $\begin{array}{r}5.54 \\
\pm 0.13 \\
\end{array}$ & $\begin{array}{r}11.68 \\
\pm 0.07 \\
\end{array}$ \\
\hline $\begin{array}{l}\text { Wheat flour type } \\
1850\end{array}$ & Marysin & $\begin{array}{r}0.130 \\
\pm 0.010\end{array}$ & $\begin{array}{r}0.417 \\
\pm 0.020\end{array}$ & $\begin{array}{r}38.48 \\
\pm 0.06\end{array}$ & $\begin{array}{r}36.04 \\
\pm 1.75\end{array}$ \\
\hline $\begin{array}{l}\text { Graham flour type } \\
1950\end{array}$ & Lublin & $\begin{array}{r}0.206 \\
\pm 0.061 \\
\end{array}$ & $\begin{array}{r}0.313 \\
\pm 0.042 \\
\end{array}$ & $\begin{array}{r}38.52 \\
\pm 3.03 \\
\end{array}$ & $\begin{array}{r}31.53 \\
\pm 1.65 \\
\end{array}$ \\
\hline \multicolumn{2}{|c|}{ mean $\pm S D$} & $\begin{array}{r}0.152 \\
\pm 0.034 \\
\end{array}$ & $\begin{array}{r}0.198 \\
\pm 0.110 \\
\end{array}$ & $\begin{array}{r}25.62 \\
\pm 9.57\end{array}$ & $\begin{array}{r}15.85 \\
+11.56 \\
\end{array}$ \\
\hline
\end{tabular}

The overall content of iron and manganese in wheat and rye flour was very similar, which correlated with the grinding. Obtained levels of chromium and nickel in rye and wheat flour were in agreement with foreign authors $[3,7,15]$ and lower in comparison to domestic results from 2005 and 2007 year $[9,10]$.

Table 4 shows the content of $\mathrm{Cr}, \mathrm{Ni}, \mathrm{Fe}$ and $\mathrm{Mn}$ in rye bread. The mean concentration of chromium equaled from $0.122 \mathrm{mg} / \mathrm{kg}$ (rye bread) to $0.237 \mathrm{mg} / \mathrm{kg}$ (grain bread) and was similar to its content in the rye flour. Nickel levels were also the lowest in rye bread $(0.073 \mathrm{mg} / \mathrm{kg})$ but the highest in mixed whole meal rye bread with grains $(0.205 \mathrm{mg} / \mathrm{kg})$, which was also comparable to Ni content in rye flour. The level of iron and manganese was the lowest in grain bread from Marysin: $15.80 \mathrm{mg} \mathrm{Fe} / \mathrm{kg}$ and $8.31 \mathrm{mg} \mathrm{Mn} / \mathrm{kg}$ respectively and the highest in mixed whole meal rye bread with grains from Lublin (21.72 $\mathrm{mg} \mathrm{Fe} / \mathrm{kg}$ and $12.11 \mathrm{mg} \mathrm{Mn} / \mathrm{kg}$ ).

Table 4. Chromium (Cr), nickel (Ni), iron (Fe) and manganese $(\mathrm{Mn})$ content in rye bread from bakery in Lublin and Marysin

\begin{tabular}{|c|c|c|c|c|c|}
\hline Product & Location & $\begin{array}{l}\mathrm{Cr}[\mathrm{mg} / \mathrm{kg}] \\
\text { mean } \pm \mathrm{SD}\end{array}$ & $\begin{array}{l}\mathrm{Ni}[\mathrm{mg} / \mathrm{kg}] \\
\text { mean } \pm \mathrm{SD}\end{array}$ & $\begin{array}{l}\mathrm{Fe}[\mathrm{mg} / \mathrm{kg}] \\
\text { mean } \pm \mathrm{SD}\end{array}$ & $\begin{array}{l}\mathrm{Mn}[\mathrm{mg} / \mathrm{kg}] \\
\text { mean } \pm \mathrm{SD}\end{array}$ \\
\hline \multirow{2}{*}{ Mixed sliced bread } & \multirow{2}{*}{ Lublin } & 0.185 & 0.154 & 19.77 & 10.21 \\
\hline & & \pm 0.036 & \pm 0.020 & \pm 0.78 & \pm 0.32 \\
\hline \multirow{2}{*}{$\begin{array}{l}\text { Mixed whole meal } \\
\text { rye bread with grains }\end{array}$} & \multirow{2}{*}{ Lublin } & 0.172 & 0.205 & 21.72 & 12.11 \\
\hline & & \pm 0.048 & \pm 0.088 & \pm 1.01 & \pm 0.31 \\
\hline \multirow{2}{*}{ Grain bread } & \multirow{2}{*}{ Marysin } & 0.237 & 0.159 & 15.80 & 8.31 \\
\hline & & \pm 0.032 & \pm 0.001 & \pm 1.27 & \pm 1.11 \\
\hline \multirow{2}{*}{ Rye bread } & \multirow{2}{*}{ Lublin } & 0.122 & 0.073 & 16.26 & 10.09 \\
\hline & & \pm 0.052 & \pm 0.037 & \pm 2.14 & \pm 0.43 \\
\hline \multirow{2}{*}{$\begin{array}{l}\text { Rye whole meal } \\
\text { bread }\end{array}$} & \multirow{2}{*}{ Marysin } & 0.157 & 0.113 & 16.11 & 9.61 \\
\hline & & \pm 0.061 & \pm 0.025 & \pm 3.45 & \pm 1.64 \\
\hline \multirow{2}{*}{\multicolumn{2}{|c|}{ mean $\pm S D$}} & 0.175 & 0.141 & 17.93 & 10.07 \\
\hline & & \pm 0.042 & \pm 0.050 & \pm 2.66 & \pm 1.37 \\
\hline
\end{tabular}

Wheat bread (Table 5) was rich in chromium at the lowest value of $0.108 \mathrm{mg} / \mathrm{kg}$ in pastoral bread and $0.316 \mathrm{mg} /$ $\mathrm{kg}$ in graham bread with spelt wheat. Nickel was observed in the same wheat bread ranging from $0.122 \mathrm{mg} / \mathrm{kg}$ (Manufacture's sliced bread) to $0.297 \mathrm{mg} / \mathrm{kg}$ in "Gwarek" bread. The content of $\mathrm{Cr}$ and $\mathrm{Ni}$ in wheat bread was similar to the level of these elements in wheat flour. Higher concentrations of named trace elements were observed in the bread with addition of extra grains, like spelt wheat or sunflower seed.
It is a result of higher content of chromium and nickel in those additives. Obtained results of chromium and nickel content in wheat and rye bread were similar to international $[3,7,15]$ and domestic $[9,10]$ authors.

Table 5. Chromium (Cr), nickel (Ni), iron (Fe) and manganese $(\mathrm{Mn})$ content in wheat bread from bakery in Lublin and Marysin

\begin{tabular}{|c|c|c|c|c|c|}
\hline Product & Location & $\begin{array}{l}\mathrm{Cr}[\mathrm{mg} / \mathrm{kg}] \\
\text { mean } \pm \mathrm{SD}\end{array}$ & $\begin{array}{l}\mathrm{Ni}[\mathrm{mg} / \mathrm{kg}] \\
\text { mean } \pm \mathrm{SD}\end{array}$ & $\begin{array}{l}\mathrm{Fe}[\mathrm{mg} / \mathrm{kg}] \\
\text { mean } \pm \mathrm{SD}\end{array}$ & $\begin{array}{c}\mathrm{Mn}[\mathrm{mg} / \mathbf{k g}] \\
\text { mean } \pm \mathrm{SD} \\
\end{array}$ \\
\hline $\begin{array}{l}\text { Mixed bread } \\
\text { "Magnus" }\end{array}$ & Lublin & $\begin{array}{r}0.181 \\
\pm 0.012 \\
\end{array}$ & $\begin{array}{r}0.167 \\
\pm 0.026 \\
\end{array}$ & $\begin{array}{r}19.63 \\
\pm 1.13\end{array}$ & $\begin{array}{r}10.41 \\
\pm 0.47\end{array}$ \\
\hline Multigrain bread & Marysin & $\begin{array}{r}0.149 \\
\pm 0.035 \\
\end{array}$ & $\begin{array}{r}0.158 \\
\pm 0.068 \\
\end{array}$ & $\begin{array}{r}15.10 \\
\pm 2.19\end{array}$ & $\begin{array}{r}7.93 \\
\pm 0.21 \\
\end{array}$ \\
\hline $\begin{array}{l}\text { Manufacture's } \\
\text { sliced bread }\end{array}$ & Marysin & $\begin{array}{r}0.245 \\
\pm 0.007\end{array}$ & $\begin{array}{r}0.122 \\
\pm 0.005\end{array}$ & $\begin{array}{r}21.36 \\
\pm 1.60\end{array}$ & $\begin{array}{r}8.56 \\
\pm 0.27\end{array}$ \\
\hline $\begin{array}{l}\text { Sunflower seed } \\
\text { bread }\end{array}$ & Lublin & $\begin{array}{r}0.195 \\
\pm 0.035 \\
\end{array}$ & $\begin{array}{r}0.250 \\
\pm 0.015 \\
\end{array}$ & $\begin{array}{r}20.08 \\
\pm 0.73 \\
\end{array}$ & $\begin{array}{r}11.99 \\
\pm 0.98 \\
\end{array}$ \\
\hline „Gwarek" bread & Marysin & $\begin{array}{r}0.191 \\
\pm 0.080 \\
\end{array}$ & $\begin{array}{r}0.297 \\
\pm 0.095 \\
\end{array}$ & $\begin{array}{r}22.27 \\
\pm 10.21 \\
\end{array}$ & $\begin{array}{r}10.10 \\
\pm 4.95 \\
\end{array}$ \\
\hline Slavic bread & Marysin & $\begin{array}{r}0.146 \\
\pm 0.057 \\
\end{array}$ & $\begin{array}{r}0.136 \\
\pm 0.083 \\
\end{array}$ & $\begin{array}{r}10.93 \\
\pm 1.45 \\
\end{array}$ & $\begin{array}{r}4.82 \\
\pm 0.58 \\
\end{array}$ \\
\hline Pastoral bread & Marysin & $\begin{array}{r}0.108 \\
\pm 0.033 \\
\end{array}$ & $\begin{array}{r}0.138 \\
\pm 0.091 \\
\end{array}$ & $\begin{array}{r}8.82 \\
\pm 3.68 \\
\end{array}$ & $\begin{array}{r}3.11 \\
\pm 1.07 \\
\end{array}$ \\
\hline $\begin{array}{l}\text { Graham bread with } \\
\text { spelt wheat }\end{array}$ & Lublin & $\begin{array}{r}0.316 \\
\pm 0.113 \\
\end{array}$ & $\begin{array}{r}0.170 \\
\pm 0.048 \\
\end{array}$ & $\begin{array}{r}18.39 \\
\pm 3.47 \\
\end{array}$ & $\begin{array}{r}9.99 \\
\pm 2.69 \\
\end{array}$ \\
\hline mean $\pm s$ & & $\begin{array}{r}0.191 \\
\pm 0.065 \\
\end{array}$ & $\begin{array}{r}0.180 \\
\pm 0.061 \\
\end{array}$ & $\begin{array}{l}17.07 \\
\pm 4.96\end{array}$ & $\begin{array}{r}8.36 \\
\pm 3.01 \\
\end{array}$ \\
\hline
\end{tabular}

Similarly to chromium, the pastoral bread from Marysin was the least rich in iron and manganese $(8.82 \mathrm{mg} \mathrm{Fe} / \mathrm{kg}$ and $3.11 \mathrm{mg} \mathrm{Mn} / \mathrm{kg}$ ) while "Gwarek" bread had the highest level of iron $(22.27 \mathrm{mg} / \mathrm{kg})$. The sunflower seed bread was the richest in manganese $(11.99 \mathrm{mg} / \mathrm{kg})$. Levels of Fe and Mn were much lower in bread than the flour from which they were baked. Additionally, wheat bread stuff had similar iron content to wheat bread ( $16.67 \mathrm{mg} \mathrm{Fe} / \mathrm{kg}$, Table 6 and $17.07 \mathrm{mg} \mathrm{Fe} / \mathrm{kg}$, Table 5 respectively).

Table 6. Chromium (Cr), nickel (Ni), iron (Fe) and manganese $\mathrm{Mn})$ content in wheat breadstuff from bakery in Lublin and Marysin

\begin{tabular}{|c|c|c|c|c|c|}
\hline Product & Location & $\begin{array}{l}\mathrm{Cr}[\mathrm{mg} / \mathrm{kg}] \\
\text { mean } \pm \text { SD }\end{array}$ & $\begin{array}{l}\mathrm{Ni}[\mathrm{mg} / \mathrm{kg}] \\
\text { mean } \pm \mathrm{SD}\end{array}$ & $\begin{array}{l}\mathrm{Fe}[\mathrm{mg} / \mathrm{kg}] \\
\text { mean } \pm \mathrm{SD}\end{array}$ & $\begin{array}{c}\mathrm{Mn}[\mathrm{mg} / \mathrm{kg}] \\
\text { mean } \pm \mathrm{SD}\end{array}$ \\
\hline \multirow{2}{*}{ Graham roll } & \multirow{2}{*}{ Lublin } & 0.173 & 0.112 & 17.08 & 6.29 \\
\hline & & \pm 0.026 & \pm 0.029 & \pm 3.92 & \pm 0.36 \\
\hline \multirow{2}{*}{ Graham roll } & \multirow{2}{*}{ Marysin } & 0.178 & 0.182 & 22.29 & 12.41 \\
\hline & & \pm 0.073 & \pm 0.034 & \pm 3.77 & \pm 2.50 \\
\hline \multirow{2}{*}{$\begin{array}{l}\text { Graham roll } \\
\text { with grain }\end{array}$} & \multirow{2}{*}{ Marysin } & 0.157 & 0.253 & 18.17 & 8.54 \\
\hline & & \pm 0.045 & \pm 0.047 & \pm 3.17 & \pm 2.23 \\
\hline \multirow{2}{*}{ Fancy roll } & \multirow{2}{*}{ Lublin } & 0.180 & 0.088 & 13.40 & 3.95 \\
\hline & & \pm 0.040 & \pm 0.015 & \pm 2.51 & \pm 0.38 \\
\hline \multirow{2}{*}{ Fancy roll } & \multirow{2}{*}{ Marysin } & 0.137 & 0.114 & 16.26 & 4.57 \\
\hline & & \pm 0.035 & \pm 0.045 & \pm 8.66 & \pm 0.32 \\
\hline \multirow{2}{*}{\begin{tabular}{|l|} 
Fancy roll \\
with powder
\end{tabular}} & \multirow{2}{*}{ Marysin } & 0.186 & 0.305 & 19.92 & 8.96 \\
\hline & & \pm 0.025 & \pm 0.004 & \pm 0.06 & \pm 0.03 \\
\hline \multirow{2}{*}{$\begin{array}{l}\text { Turkish roll } \\
\text { with raisins }\end{array}$} & \multirow{2}{*}{ Marysin } & 0.131 & 0.093 & 13.62 & 4.12 \\
\hline & & \pm 0.022 & \pm 0.022 & \pm 1.25 & \pm 0.66 \\
\hline \multirow{2}{*}{ Sweet Turkish roll } & \multirow{2}{*}{ Marysin } & 0.182 & 0.103 & 12.58 & 3.38 \\
\hline & & \pm 0.009 & \pm 0.022 & \pm 1.15 & \pm 0.18 \\
\hline \multirow{2}{*}{\multicolumn{2}{|c|}{ mean $\pm S D$}} & 0.166 & 0.156 & 16.67 & 6.53 \\
\hline & & \pm 0.021 & \pm 0.082 & \pm 3.41 & \pm 3.18 \\
\hline
\end{tabular}

Taking into account a large amount of water in bread comparing flour from which they were obtained it could be suggested that similar $\mathrm{Cr}$ and $\mathrm{Ni}$ contents in bread and flour point out to contamination of bread by these trace elements during their production. Other authors [1] also observed this phenomenon.

In wheat bread stuff (Table 6) mean chromium and nickel concentrations were $0.166 \mathrm{mg} / \mathrm{kg}$, and $0.156 \mathrm{mg} / \mathrm{kg}$ respectively. These levels were a little bit smaller than in wheat bread and similar to the content of elements in the flour, which indicates that chromium and nickel in breadstuff could be an effect of impureness during technological 
process of their production. Similar observations were reported by Brügemann [3] and Alomary [1].

Relatively high contents of analyzed elements were observed in ready-prepared mixture „Gwarek” used for baking of „Gwarek” bread, which contained $0.312 \mathrm{mg} \mathrm{Cr} / \mathrm{kg}$, $0.570 \mathrm{mg} \mathrm{Ni} / \mathrm{kg}$ and $45.66 \mathrm{mg} \mathrm{Fe} / \mathrm{kg}$ (Tab. 7).

Table 7. Chromium (Cr), nickel (Ni), iron (Fe) and manganese $(\mathrm{Mn})$ content in "gwarek" mixture and wheat bran from bakery in Marysin

\begin{tabular}{|l|l|c|c|c|c|}
\hline \multicolumn{1}{|c|}{ Product } & \multirow{2}{*}{ Location } & $\begin{array}{c}\mathrm{Cr}[\mathrm{mg} / \mathrm{kg}] \\
\mathrm{mean} \pm \mathrm{SD}\end{array}$ & $\begin{array}{c}\mathrm{Ni}[\mathrm{mg} / \mathrm{kg}] \\
\text { mean } \pm \mathrm{SD}\end{array}$ & $\begin{array}{c}\text { Fe }[\mathrm{mg} / \mathrm{kg}] \\
\text { mean } \pm \mathrm{SD}\end{array}$ & $\begin{array}{c}\mathrm{Mn}[\mathrm{mg} / \mathrm{kg}] \\
\text { mean } \pm \mathrm{SD}\end{array}$ \\
\hline \multirow{2}{*}{ "Gwarek" mixture } & \multirow{2}{*}{ Marysin } & 0.312 & 0.570 & 45.66 & 18.53 \\
& & \pm 0.035 & \pm 0.041 & \pm 0.64 & \pm 1.48 \\
\hline \multirow{2}{*}{ Wheat bran } & \multirow{2}{*}{ Marysin } & 0.174 & 0.237 & 41.39 & 32.95 \\
& & \pm .029 & \pm 0.015 & \pm 5.47 & \pm 4.97 \\
\hline
\end{tabular}

While comparing all samples, the chromium concentration was similar in three groups of products: rye and wheat flour and breadstuff. Significantly higher level of chromium was found in "Gwarek" mixture and in graham bread with spelt wheat (Fig. 1 and 3). The highest concentration of nickel was observed in "Gwarek" mixture, wheat flour with high level of grinding (type 1850 and 1950) as well as in sunflower seed bread and "Gwarek" bread ( Fig. 1 and 3). Wheat bran and rye flour showed the highest content of manganese, which correlated with the grinding. Similarly, iron content also dependent on grinding was the highest in "Gwarek" mixture, bran and rye flour (Fig. 2 and 4).

Our results are in agreement with the foreign researchers from Finland [15], Germany [3] and Sweden [7] and very similar to the previous results obtained in grains and cereal goods performed in the Chair and Department of Food and Nutrition (Medical University of Lublin) in 2004 [8] and 2006 [9].

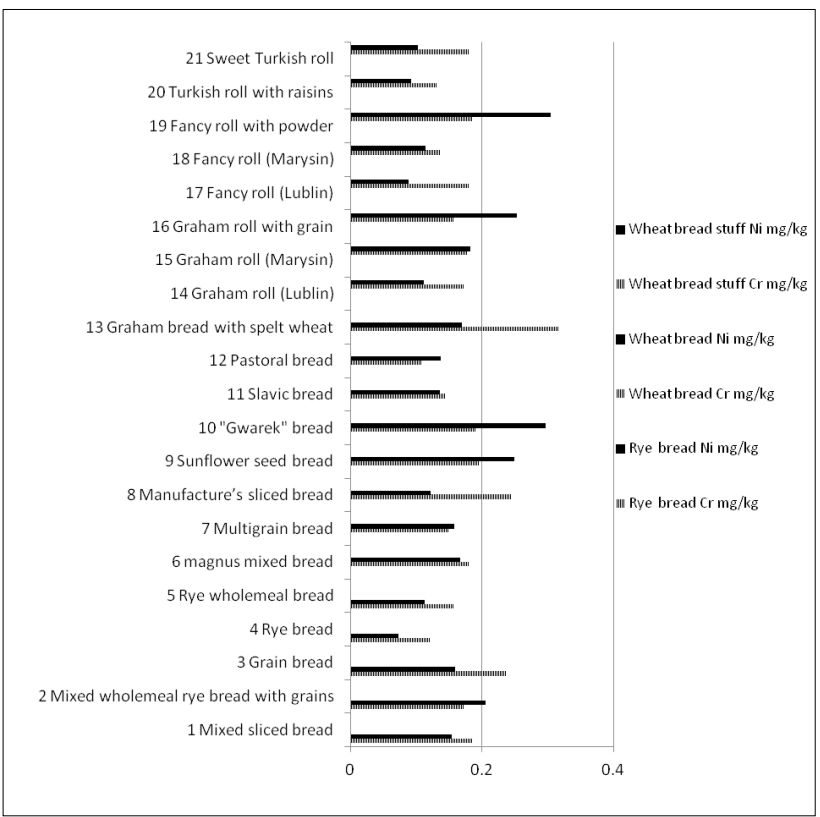

Figure 1. Chromium $(\mathrm{Cr})$ and nickel $(\mathrm{Ni})$ content in bread stuff and bread from bakery in Lublin and Marysin

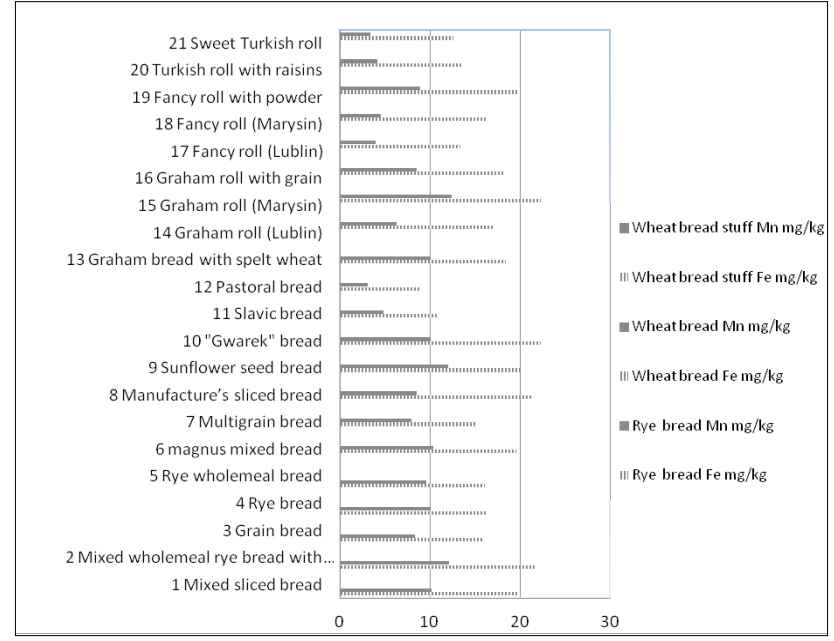

Figure 2. Iron ( $\mathrm{Fe}$ ) and manganese ( $\mathrm{Mn})$ content in breadstuff and bread from bakery in Lublin and Marysin

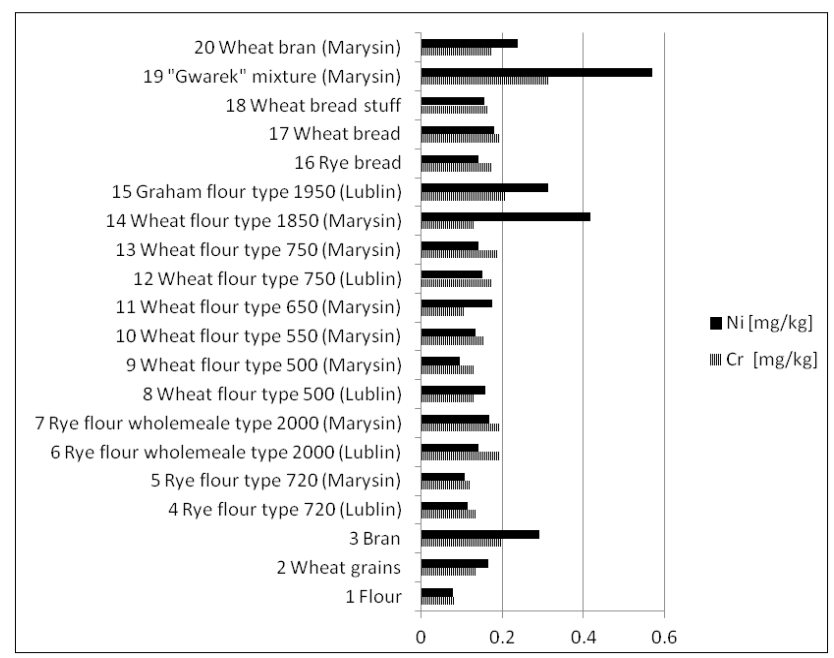

Figure 3. Chromium $(\mathrm{Cr})$ and nickel $(\mathrm{Ni})$ content in grains, bran, flour and breadstuff flour from mill and bakeries

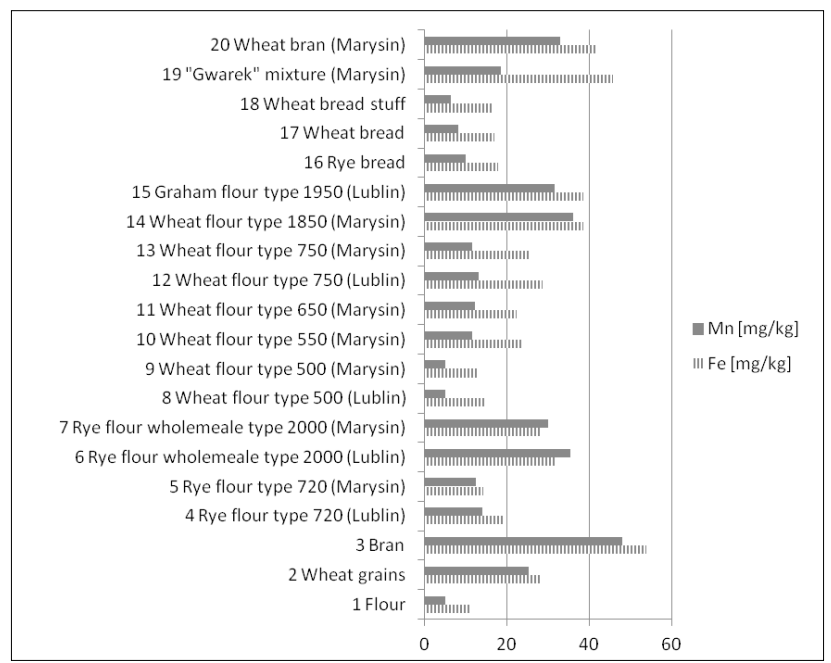

Figure 4. Iron (Fe) and manganese ( $\mathrm{Mn})$ content in grains, bran, flour and breadstuff flour from mill and bakeries 


\section{CONCLUSIONS}

Bread and grain products are a good source of trace elements like chromium, nickel, iron and manganese. The concentrations of those elements were observed in grain products enriched in sunflower seeds, spelt wheat and wheat grains. However, the higher levels of chromium and nickel in bread goods could be also an effect of impurity caused by a technological process, which was not observed in the case of iron and manganese.

\section{REFERENCES}

1. Alomary A. Wedian F.: The Influence of Baking Fuel Types on the Residues of Some Heavy Metals in Jordanian Bread. JJC, 7(1), 2012.

2. Ambroziak Z.: Piekarstwo i ciastkarstwo, Wydawnictwo Naukowo Techniczne, Warszawa 1988.

3. Brüggemann J., Dorfner H. H., Heckt H. et al.: Status of Trace Elements in Staple Foods from Germany 1990-1994. FAO REH Technical Series 49, 1998.

4. Concise Statistical Yearbook of Poland 2012.

5. Grembecka M., Kusiuk A., Szefer P.: Content of magnesium, phosphorus, zinc and iron in different kinds of bread. Bromat. Chem. Toksykol., 4, 2007.
6. Isserliyska D., Karadjov G., Angelov A.: Mineral composition of Bulgarian wheat bread. Eur. Food Res. Technol., 213, 2001.

7. Jorhem L.: Cadmium and Other Metals In Swedish wheat and Rye Flours. Longitudial Study, JAOAC Int., 84, 2001.

8. Kot A., Zaręba S.: Produkty zbożowe źródłem żelaza i manganu, Roczn. PZH, 56(1), 2005.

9. Kot A.: Chromium and Nickel Content in Cereal Products. Polish J. Environ. Stud. Vol. 16, 3A, 2007.

10. Kot A.: Produkty zbożowe źródłem niklu, Nowiny Lek. 74(4), 2005.

11. Maruszewska M., Bolesławska J., Przysławski J.: Preferencje osób dorosłych w zakresie spożycia przetworów zbożowych, Sympozjum Bezpieczna żywność i prawidłowe odżywianie podstawą profilaktyki zdrowotnej, Wrocław, 19-20 września 2002.

12. Nikonorow M., Urbanek - Karłowska B.: Toksykologia żywności PZWL, 1987. [5]

13. Sykuła-Zając A., Pawlak A.: Chromium in food products. Biotechnol Food Sci. 76 (1), 2012.

14. Szczęsna T., Wojtala M., Waszkowiak K.: The effect of nutritional knowledge, education and financial situation on food preferences and nutritional habits of agricultural university students living in students house. Probl. Hig. Epidemiol. 86 (1), 2005.

15. Varo P., Nuurtamo E., Saart E., et al.: Mineral Element Composition of Finish Foods, IV, Flours and Bakery Products, Acta Agricultuare Scandinavica, Suppl. 22, 1980. 